\title{
Relativistic Stars in dRGT Massive Gravity ${ }^{\dagger}$
}

\author{
Masashi Yamazaki 1,*, Taishi Katsuragawa ${ }^{2, *}$, Shin'ichi Nojiri ${ }^{1,3,4, *}$ and Sergei D. Odintsov 5,6,* \\ 1 Department of Physics, Nagoya University, Nagoya 464-8602, Japan \\ 2 Institute of Astrophysics, Central China Normal University, Wuhan 430079, China \\ 3 Kobayashi-Maskawa Institute for the Origin of Particles and the Universe, \\ Nagoya University, Nagoya 464-8602, Japan \\ 4 KEK Theory Center, High Energy Accelerator Research Organization (KEK), Oho 1-1, Tsukuba, \\ Ibaraki 305-0801, Japan \\ 5 ICREA, Passeig Luis Companys, 23, 08010 Barcelona, Spain \\ 6 Institute of Space Sciences (IEEC-CSIC) C. Can Magrans s/n, 08193 Barcelona, Spain \\ * Correspondence: yamazaki.masashi@c.mbox.nagoya-u.ac.jp (M.Y); taishi@th.phys.nagoya-u.ac.jp (T.K.); \\ nojiri@gravity.phys.nagoya-u.ac.jp (S.N.); odintsov@ieec.uab.es (S.D.O.) \\ † Presented at Symmetry 2017-The First International Conference on Symmetry, Barcelona, Spain, \\ 16-18 October 2017.
}

Published: 4 January 2018

The de Rham-Gabadadze-Tolley (dRGT) massive gravity describes a massive spin-2 particle. The theory is one of modified gravities, where cosmological behaviors change from those in general relativity. In order for the massive gravity theory to provide solar system observations, in short-range scale, the theory needs to show similar behaviors to those in general relativity. The behaviors can be obtained by the Vainshtein mechanism, where the non-linear kinetic terms in the dRGT massive gravity allow an extra degree of freedom in the theory. Although the mechanism has only been verified in a vacuum in static and spherically symmetric configurations, such as a point-like particle, it is not apparent whether the Vainshtein mechanism could work if matter exists in non-static or non-spherical configurations.

In the environment of relativistic stars, the gravity is strong and the density of the matter is high, which could lead to non-trivial behaviors in the Vainshtein mechanism. In our research, we construct solutions describing static and spherical relativistic stars in the minimal model of the theory. A new equation, derived from consistency conditions, determines the behavior of the extra degree of freedom. By numerical calculation, we show that the maximal mass in massive gravity is smaller than that in general relativity for several equations of state. The results could be consistent with previous studies on the energy scales in which non-linear kinetic terms appear. Moreover, we report the studies of relativistic stars in the non-minimal model of the theory.

(C) 2018 by the authors. Licensee MDPI, Basel, Switzerland. This article is an open access article distributed under the terms and conditions of the Creative Commons Attribution (CC BY) license (http://creativecommons.org/licenses/by/4.0/). 